\title{
Behavior and Techniques for Improving Performance of OFDM Systems for Wireless communications
}

\author{
Alcardo Alex Barakabitze ${ }^{1}$, Md. Abbas Ali ${ }^{2}$ \\ School of Communication Engineering, Chongqing University, China ${ }^{1,2}$
}

\begin{abstract}
Orthogonal frequency division multiplexing (OFDM) is a special case of multicarrier transmission which transmits a stream of data over a number of lower data rate subcarriers. OFDM splits the total transmission bandwidth into a number of orthogonal and non-overlapping subcarriers and transmit the collection of bits called symbols in parallel using these subcarriers. This paper gives a total insight of various Peak -to Average Power Reduction (PAPR) techniques and principles of OFDM systems used in wireless communications. The research paper places a focus also on OFDM behaviors and techniques like Carrier Frequency Offset (CFO) estimation that improves performance of OFDM for wireless communications. Finally, the paper provides a number of wireless communication standards and many of the applications where OFDM systems are used.
\end{abstract}

Keywords: OFDM, Peak-to-Average Power Reduction (PAPR), Inter-Carrier Interference (ICI), Channel estimation, Cyclic Prefix, Bit Error Ratio (BER).

\section{INTRODUCTION}

The recent years has witnessed the demand for higher data and power efficiency[3]. OFDM is a special form of rate services such as voice, data and multimedia over both Multi-Carrier Modulation (MCM) technique that was wired and wireless communication links [1]. This demand developed in early 1960s [13]. MCM involves splitting the has increased due to the rapid growth of digital input signal to a number of different signals over one communication systems [2]. The wired and wireless frequency range. Each of these signals are then communication environments has been requiring new transmitted, modulated and demodulated over the channel. technologies with new applications like the mobile web Several high-frequency military systems such as access, IP telephony, gaming services, high-definition KINEPLEX [15], ANDEFT [16], and KATHRYN [17] mobile TV, video conferencing,3D television and cloud started to use the OFDM technique in 1960s where by the computing[109]. The mobile communication systems like variable-rate data modem was built for the high-frequency 4G and 5G are expected to provide substantially high data band (i.e., in KATHRYN) which used up to 34 parallel rate transmissions in order to meet the requirements of low-rate phase-modulated channels with a sub-carrier future high performance services such as multimedia, spacing of $82 \mathrm{~Hz}$. Since its development in early 1960s, voice, and data applications. For example, in March 2008, OFDM was then proposed tobe used in wireless the International Telecommunications Union-Radio applications in 1980s [14]. Due to its reasonable prices of communications sector (ITU-R) set requirements hardware implementations which rely on a very high specification of $4 \mathrm{G}$ standards called the International speed digital signal processing, OFDM has been Mobile Telecommunications Advanced (IMT-Advanced) recognized as an outstanding technique for high-speed specification [9].The ITU-R set a maximum speed for 4G data communication systems (e.g. 4G, 5G, LTEservice at $100 \mathrm{Mbps}$ for high mobility communication (i.e. Advanced).In order to maintain a high data rate from trains and cars) and 1Gbps for low mobility transmission, OFDM takes and divides the total available communication such as pedestrians and stationary bandwidth into a number of sub-carriers which are then users[9].

However, the current $4 \mathrm{G}$ communication system suffers from some common problems such as attenuation, multipath fading, delay spread, self-jamming.etc[11]. New techniques and solutions are required to be embedded in $4 \mathrm{G}$ and $5 \mathrm{G}$ communication systems in order to eliminate and solve the problems which can hinder the performance of communications. OFDM is a promising candidate technology and a modulation technique which is used to provide such a high data rate in the emerging mobile communication systems like 4G and 5G.OFDM in the 4G and $5 \mathrm{G}$ community, provide high data rate transmissions with high spectral efficiency, multipath delay speed transmitted in parallel from the transmitter to the receiver through a channel. Every sub-carrier is then modulated by a low rate data stream. On reaching to the receiver, these sub-carriers are combined together so as to form a composite high-data rate communication system. The subcarriers in OFDM systems are orthogonal to each other and this orthogonality condition enables the sub-carriers to be closely spaced and overlap without Inter-CarrierInterference (ICI).

Although FDMA,TDMA, CDMA are the well-known multiplexing techniques used in wireless communication systems over a number of years, the overall problems tolerance, immunity to frequency selective fading channels Interference (ISI) resulted from time dispersion, multipath 
fading, less spectral efficiency and the requirement of performance of OFDM systems such PAPR reduction and larger transmitting power for high-bit rate. The above Carrier Frequency Offset (CFO) estimation techniques mentioned problems can be solved by using OFDM [3][4][31][67], but still there is a high need of contributing modulation techniques. OFDM is a very powerful a comprehensive survey regarding to principles, PAPR modulation technique which is capable of achieving high reduction techniques, CFO estimation methods as well as data rate transmission without ISI and Inter Carrier applications of OFDM through a theoretical analysis. In Interference (ICI). OFDM is made more efficient by the fact, CFO estimation and PAPR techniques should be use of IFFT and its inverse FFT techniques to given the first priority and a research trade-off through implementmodulation and demodulation functions performance criterion such as, power increase in the respectively. The OFDM based wireless communication transmit signal, PAPR reduction capability, data rate loss, system design includes the design of OFDM transmitter, implementation complexity, Bit-Error-Ratio (BER) etc.

and OFDM receiver.

An algorithm in OFDM systems is adopted in the process of generating and receiving sub-carriers by using the digital signal processing technology. However, in order to improve frequency spectrum utilization, each sub-carrier spectrum which meet the orthogonality condition throughout the symbol period(which is longer with narrower bandwidth compared to single-carrier research directions.

modulation) is overlapped to ensure the receiving end recover the signal without distortion. The OFDM signal spectrum is shown in Figure 1.

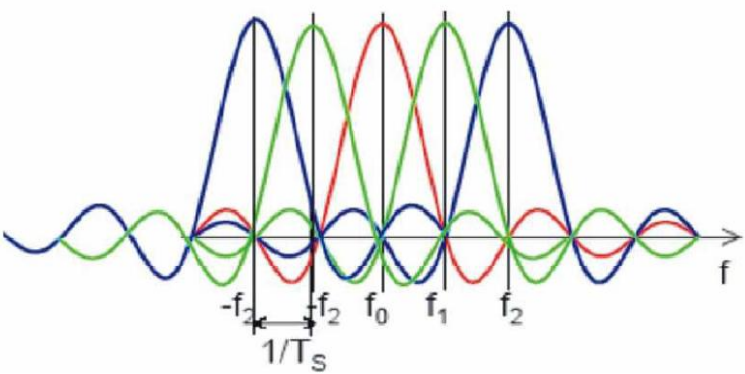

The rest of this paper is organized as follows: Section II provides the basic principle of OFDM systems, section III describes in more detail different techniques for improving the performance of OFDM for wireless communications, and section IV highlights the applicability of OFDM in many wireless communication standards and applications.

\section{Basic Principle of OFDM System}

The basic principle of OFDM is to split a high-rate data stream into a number of lower rate streams that aretransmitted simultaneously in parallel over a number of subcarriers. In order to allow for a high spectral efficiency in OFDM systems, the modulation scheme to be used and the carrier power are controlled individually for each carrier. OFDM is generated by choosing the spectrum required based on the input data (stream of bits), and modulation scheme to be used. All the sub-carriers are carefully controlled in order to maintain the orthogonality condition between the carriers. Every sub-carrier OFDM

Despite having a number of literature survey papers concerning OFDM, techniques for improving the systems is assigned the same data during transmission.

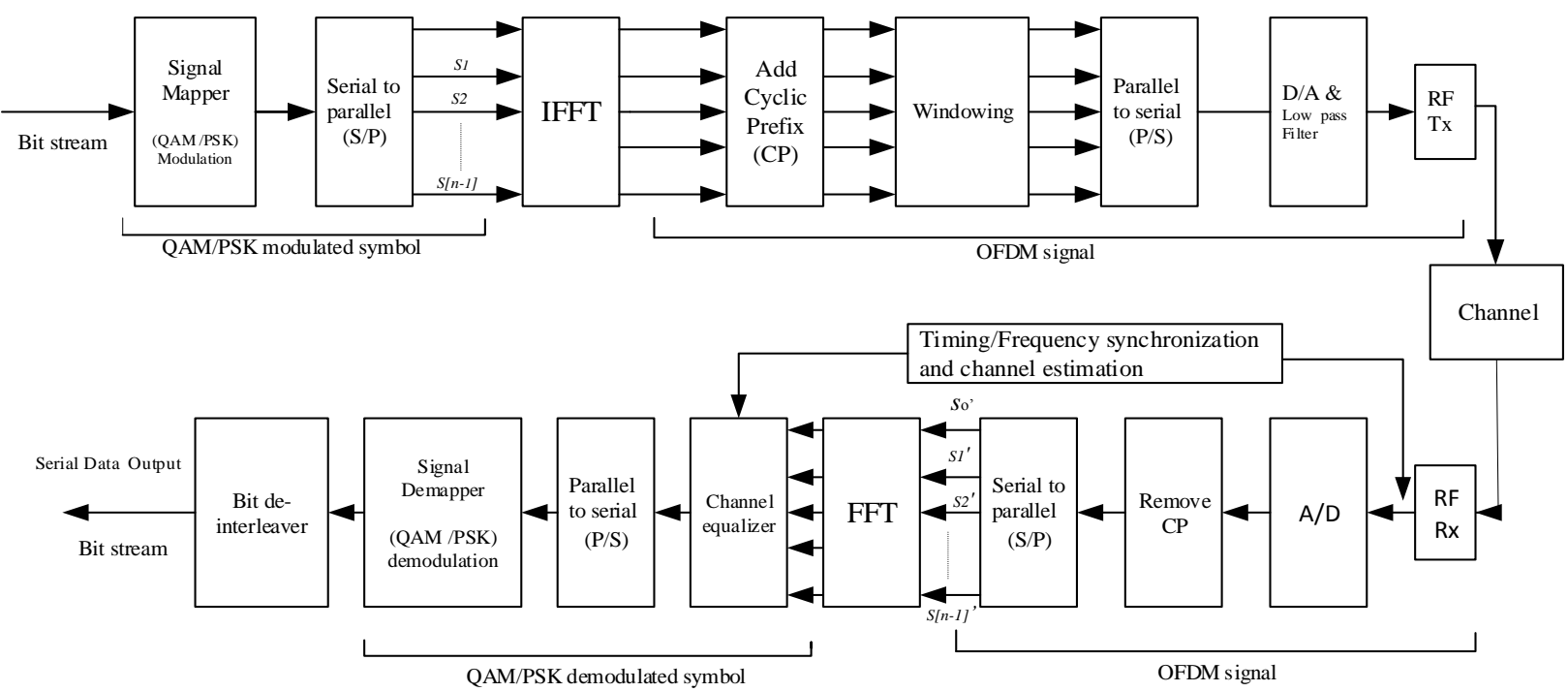

Figure 2: OFDM system block diagram

As shown in Fig 2, a binary message in form of bits at the channel conditions and the need of getting the best balance transmitter is generated which has a length divisible by the between the Bit Error Rate (BER) and frequency spectrum number of subcarriers. The signal of each sub-carrier is utilization. The input serial stream of data is then first modulated. Each sub-carrier in OFDM system can converted to parallel sets of data at the transmitter. Each adopt different modulation scheme such as BPSK, QPSK, set of data in OFDM systems contains one symbol say $S n$ 8PSK, 16QAM, 64QAM, etc. This is so depending on the 
for every subcarrier. Taking an example, a set of four data set would be [SO, S1, S2,S3]. Figure 3 shows how these

data set is arranged in the horizontal axis in the frequency domain.

The frequency distribution domain of symbols takes the symmetrical arrangement about the vertical axis. This arrangement is necessary for using the Inverse FFT in order to convert the frequency domain data sets into sample data of the corresponding time domain representation. In OFDM, the IFFT is useful for OFDM system due to the fact that, the samples of a waveform are generated with orthogonal frequency components. A Cyclic Prefix (CP) is added to each symbol followed by windowing which is a popular method of reducing spectral side lobes in OFDM. Then, the parallel to serial block creates the OFDM signal by sequentially outputting the time domain samples followed also by digital to analog conversion. CP is a periodic extension of the last part of an OFDM symbol.

The CP is added to the front of the symbol in the transmitter and then removed at the receiver before the demodulation process is performed. The $\mathrm{CP}$ is inserted in order to convert the linear convolution with the channel impulse response into a cyclic convolution. The cyclic convolution then results into a diagonalised channel that becomes free of both ISI and ICI interference. The CP acts as a guard space between successive OFDM symbols and therefore prevents Inter - Symbol Interference (ISI) as long as the length of the CP is longer than the impulse response of the channel. The Cyclic Prefix also ensures orthogonality between the sub-carriers by keeping the OFDM symbol periodic over the extended symbol duration, and therefore avoiding Inter-Carrier Interference (ICI).

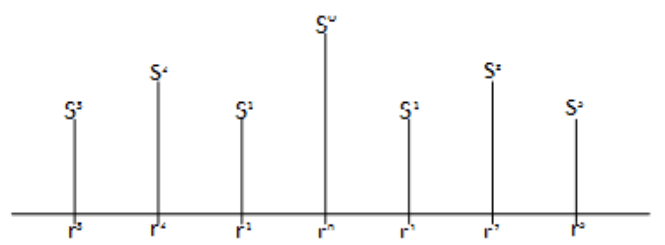

Figure 3: Frequency Domain Distribution of Symbol

The original data is then recovered from the modulated signal through OFDM demodulationtechnique. The received signal pass through a low pass filter and the guard cyclic prefix is removed. The serial data stream is then converted to parallel followed by the FFT of the signal. A demodulator is then used to get back the original signal. By taking the considerations of the un-modulated signal and the data at the receiving end, the bit error rate and the signal to noise ratio is calculated.

However, OFDM is constrained by a number of problems also including the Peak-to average power ratio (PAPR), time and frequency synchronization errors which are resulted from the mismatch of oscillators or from the doppler spread due to relative movement between the receiver and transmitter. Time and frequency varying wireless channels affect the performance of OFDM systems. Time-varying impairments is the result of theCarrier Frequency Offset (CFO) caused by the mismatch of frequencies between the oscillators at the receiver and transmitter. Frequency-varying impairments is the result of the timing offset or the delay spread of wireless channels [3].Although OFDM systems are more robust to frequency-selective fading channels but they are more sensitive to the time-frequency varying impairments of channels. These impairments can be completely cancelled in time domain through CFO estimations of which this paper places a focus. TheICI between subcarriers in OFDM systems is introduced by CFO which then destroy the sub-carriers orthogonality and therefore resulting into degradation of the bit error rate (BER).CFO also introduces a phase change proportional to OFDM symbol number as well as the cyclic shift of data between sub-carriers. This paper focus on different PAPR reduction techniques in OFDM systems which ultimately improves the performance of wireless communications. The effects of CFO in OFDM systems will be also covered in a quantitative manner. The significance is to correct this CFO and timing offset or the delay spread at the receiver such that the performance of OFDM systems over a wireless communication channel can be significantly improved.I

\section{TECHNIQUES TO IMPROVE THE PERFORMANCE OF OFDM FOR WIRELESS COMMUNICATIONS.}

In order to realize the efficiency of OFDM system, it is necessary to justify the performance using different techniques and affecting factors like channel noise, multipath delay spread e.t.c. Techniques to improve the performance of OFDM for wireless communications include Peak-to-Average Power Ratio (PAPR) reduction techniques, channel estimation, time- and frequencyvarying impairment mitigation techniques e.t.c. The motivation for PAPR reduction in OFDM systems has been shown in [68] to be the nonlinear characteristics of High Power Amplifier (HPA) and Digital-to-Analog Converter (DAC) that can degrade system performance due to induced spectral regrowth and detection inefficiency. Another motivation is the power saving, since due to the fact that, when the HPA have a high dynamic range, then the power efficiency tends to be poor [68]. Baxley and Zhou [69] have indicated that, the PAPR reduction in OFDM systems can save the power significantly. Theyfurther stress that, the desired average output power is directly proportional to the net power saving and highly depends on the level of clipping probability.

\section{A. Peak-to-Average Power Ratio (PAPR)} reduction techniques

High Peak-to-Average Power Ratio has been recognized as one of the major practical problem involving OFDM modulation. High PAPR results from the nature of the modulation itself where multiple subcarriers or sinusoids are added together to form the signal to be transmitted. PAPR is defined as the ratio of the maximum instantaneous power and the average power,i.e Maximum 
power occurring in the OFDM transmission to the average power of the OFDM transmission. Mathematically defined as $\mathrm{P}_{\text {peak }} / \mathrm{P}_{\text {average }}$

$$
P A P R=\underset{0 \leq t \leq T}{\operatorname{Max}} \frac{[x(t)]^{2}}{E\left\{|x(t)|^{2}\right\} \mid}
$$

WhereE $\{\bullet\}$ Denotes expectation operator.For example, an OFDM signal with a total of 128 carriers each with normalized power of $1 \mathrm{~W}$, then the maximum PAPR can be as large as $10 \log 10128$ or $21 \mathrm{db}$. This is at the instant when all 128 carriers combine at their maximum point which is unlikely but possible.

In order to improve the performance of OFDM in wireless communication systems, several techniques have been proposed in the literature to reduce the PAPR. These techniques are mainly categorized in two parts namely:

\section{a) Signal scrambling techniques \\ b) Signal distortion techniques}

Signal scrambling techniques involve the process of scrambling an input data block of OFDM symbols and transmit the one with a minimum PAPR. In this case, the probability of incurring high PAPR is also significantly reduced [42].These techniques are further sub-divided into two categories that is, techniques which requires explicit side information and techniques which does not need explicit side information. Signal scrambling techniques with side information includes Block Coding Techniques [20][21], Selected mapping (SLM)[22][23][24][57], ,PartialTransmit Sequence (PTS)[25-28][58], Interleaving Technique[42], Tone Reservation (TR)[54][62], Tone Injection(TI)[54] and constellation extension techniques[29-30].Although the coding technique minimizes the PAPR and causes no distortion as well as creating no out-of-band radiation, but as the code rate is reduced this technique suffers from bandwidth efficiency. Another drawback of this technique is its complexity which is encountered when finding the best codes as well as when storing large lookup tables for encoding and decoding when a large number of sub-carriers are used [31]. Among the approaches used as signal scrambling techniques are theGolay complementary sequence, Reed Muller code, M-sequence, Barker codes and Hadamard code [32-41].However, the Signal scrambling techniques with side information reduces the effective throughput since they introduce redundancy.

The signal distortion techniques involves the nonlinear saturation around the peaks in order to reduce the PAPR in OFDM systems [42]. According to Vijayarangan and Sukanesh[43], the signal distortion techniques can directly reduce high peaks through signal distortion before performing signal amplification. Although this technique for reducing PAPR is simple to implement but it can cause both in-band and out-of-band interferences, complexity to the system as well as destroying the orthogonality among the sub-carriers [42][43].In this case, the system, performance can be greatly degraded. The practical Copyright to IJARCCE approaches for signal distortion techniques includes clipping and filtering technique [44-46][48],Active constellation [47][30],Peak Windowing techniques[49],Envelope Scaling[50], Random phase updating[51], Peak reduction carrier[52], Companding[54][59], Fourier projection technique, and decision-aided reconstruction technique[49][55][56].Other PAPR techniques includes the adaptive pre-distortion and the DFT-spreading techniques. In the adaptive predistortion technique, the nonlinear effect and time variations of the High Power Amplifiers in OFDM systems is compensated [60].However, through the use of broadcasting technique as well as designing appropriate training signals then, the Maximum Square Error (MSE) and the convergence time of the adaptive pre-distorter is reduced significantly [42].In the DFT-spreadingtechnique, the input signal is first speeded with the DFT and subsequently taken into IFFT where the PAPR of OFDM signal is minimized or reduced to the level of singlecarrier transmission[42]. The DFT spreading technique is very applicable in the up-link transmission of a mobile terminal and is well known as the Single Carrier-FDMA (SC-FDMA) which has also been adopted and applied for 3GPP LTE standard uplink transmission[63-66].

Among the factors for selecting the best PAPR reduction techniques includes high capability of PAPR reduction by avoiding the harmful side effects that can result into the in-band distortion and out-of-band radiation. Another factors of concern are the data rate loss, implementation complexity, Bit-Error-Ratio (BER) and so on. In this paper, we mainly describe three practical PAPR reduction techniques which are Clipping and Filtering, Selective Mapping and the Partial Transmit Sequence.

\section{i. $\quad$ Selective Mapping Technique(SLM)}

Fig 4 shows the block diagram of the selective mapping technique for PAPR reduction in OFDM systems. In SLM approach, the input data block $X=x[0], x[1], x[2] \ldots \ldots . . x[N-1]$ to be transmitted is multiplied by $\mathrm{V}$ different phase sequences $P^{v}=\left[p_{0}^{v}, p_{1}^{v}, p_{2}^{v} \ldots \ldots p_{N-1}^{v}\right] \quad$ where $\mathrm{V}=[0,1,2 \ldots \mathrm{v}-1]$ such that the resulting data block becomes of sequence $X^{v}=\left[x^{v}[0], x^{v}[1] \ldots \ldots x^{v}[N-1]\right]^{T} \quad$ and convertsinto OFDM signals by the inverse FFTs. The signal with the minimum PAPR is then selected for final serial transmission [42].In fact, when the receiver becomes aware of the predetermined sequence which will enables the minimum PAPR then, the transmitted symbols $X^{v}$ can be easily recovered[3]. In SLM, the predetermined book sequences $X^{v}=\left[x^{v}[0], x^{v}[1] \ldots \ldots x^{v}[N-1]\right]^{T}$ are known by both, the transmitter and the receiver. Therefore, the information index of the predetermined sequence $P^{v}$ 
needs to be sent to the receiver for each OFDM signal as a SLM implementation requires V IFFT operations and side information [31]. However, in order to improve the transmission efficiency, transmission of side informationcan be omitted by embedding it into an OFDM signal, for example, through a label inserted scrambler and an encoder, or by blindly detecting the optimal phase sequence index in the receiver [3].In OFDM systems, the needs also $\left[\log _{2} V\right]$ bits of side information for each data[3][42][68].In order to reduce the computational complexity imposed in SLM, some extension of this techniques has been proposed as shown in [68] and the work of Breiling et.al [23] which performs PAPR reduction without the need of side information.

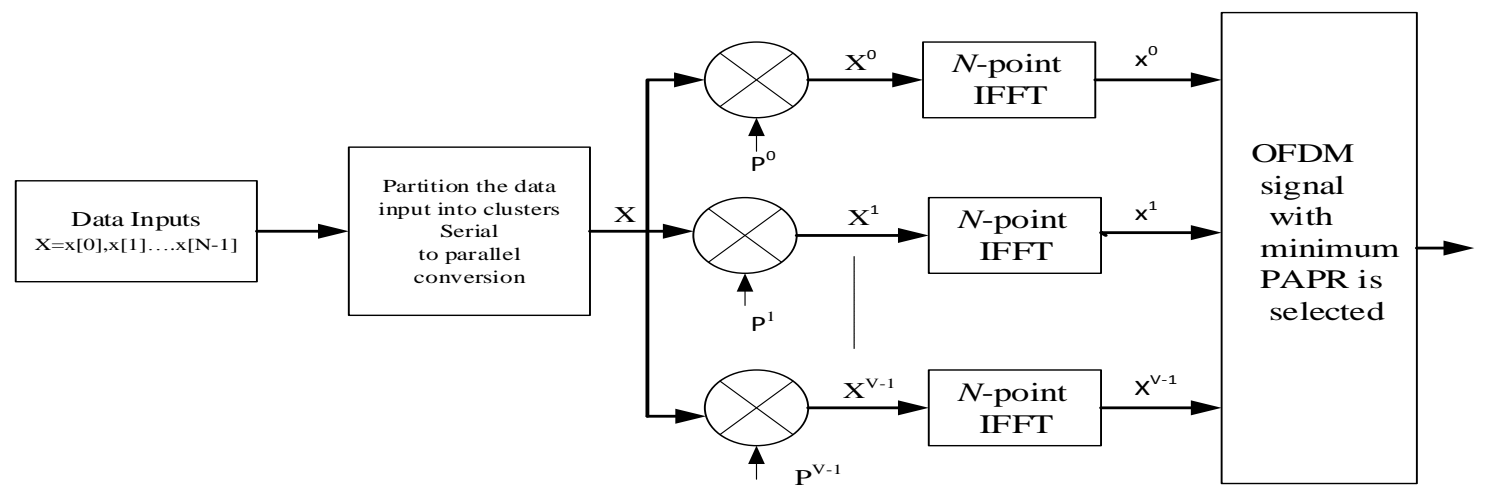

Fig 4: Block diagram of selective mapping (SLM) technique for PAPR reduction.

\section{ii. $\quad$ Partial Transmit Sequence(PTS)}

The PTS approach is based on partitioning the transmitted OFDM data blocks into several sub-blocks and for each sub-block, multiplying by different weight factors until an optimum value is achieved [42]. As shown in Fig 5, the data information in frequency domain $X$ of $\mathrm{N}$ symbolsis separated into Vnon-overlapping sub-blocks and each subblock vectors has the same size $X=\left[x^{0}, x^{1}, x^{2} \ldots . . x^{V-1}\right]^{T}$ Where $X^{i}$ represents the sub-blocks that are located consecutively to each other.Every partitioned sub-block is then multiplied by its corresponding complex phase factor such that $b^{v}=e^{j \varphi v}$ where $\mathrm{v}=1,2,3 \ldots . \mathrm{V}$. In order to obtain the signal in time domain, the IFFT operation is performed on $X^{i}$ to yield the following

$X=\operatorname{IFFT}\left\{\sum_{v=1}^{v} b^{v} X^{v}\right\}=\sum_{v=1}^{v} b^{v} \cdot \operatorname{IFFT}\left\{X^{v}\right\}=\sum_{v=1}^{v} b^{v} X^{v}$ Where $\left\{X^{v}\right\}$ is the PTS. In the next step, a suitable factorcombination that is $b=\left[b^{1}, b^{2}, b^{3} \ldots \ldots b^{v}\right]$ is made in the process of finding the minimum value suitable for reducing PAPR in OFDM systems.

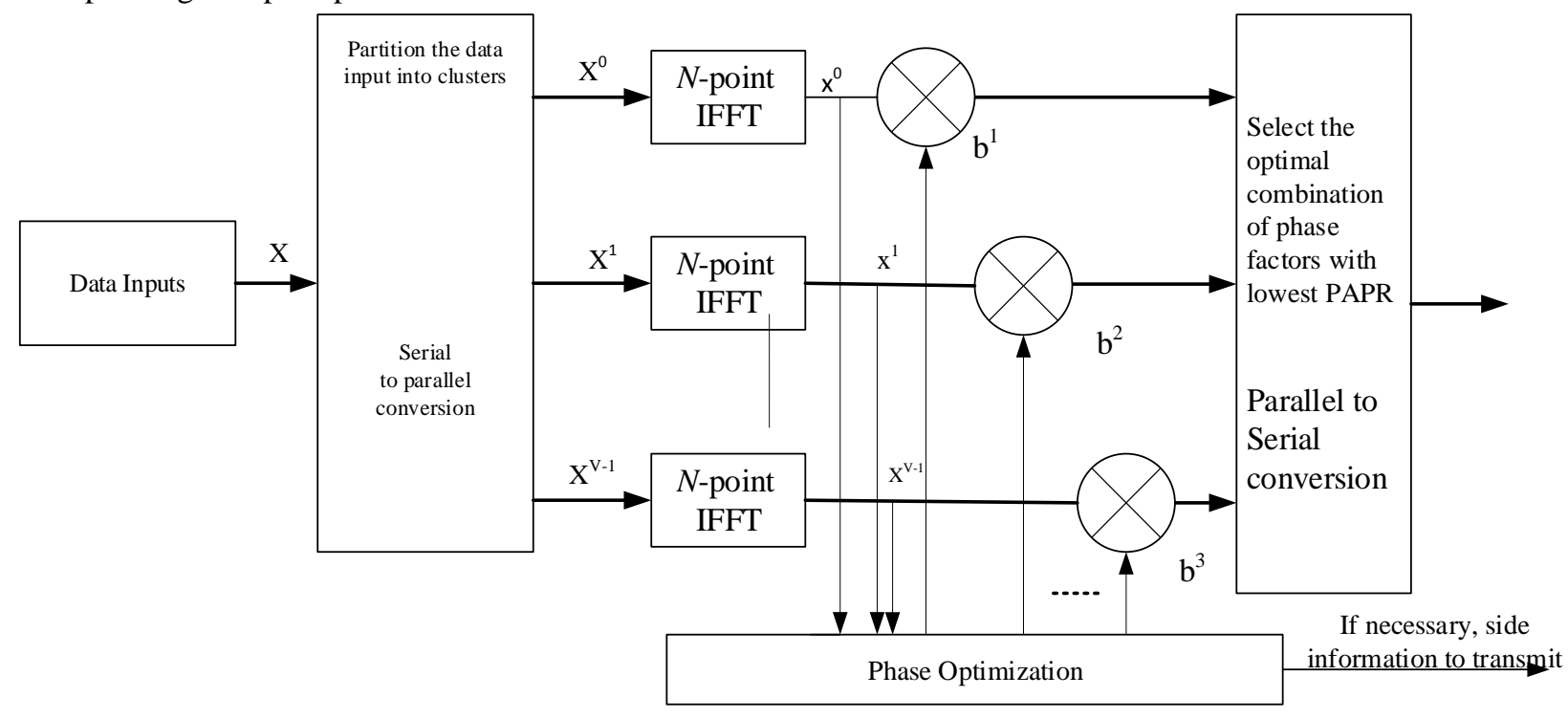

Fig 5: Block diagram of PTS algorithm for reducing PAPR 
However, the PTS suffers from two main issues which areiii. - High computational complexity which is resulted while searching the optimal phase factors.

- The overhead of the optimal phase factors incurred as a side information required to be transmitted to receiver for the correct decoding of the transmitted OFDM symbols.

In order to reduce searching complexity and the use of side information, several extensions of PTS has been proposed recently in the literature [69-77]. In [74], a Genetic algorithm based PTSmethod (GA-PTS) is proposed as a novel and sub-optimal PAPR reduction. The GA-PTS has lower computational load than original PTS method since it takes advantages of genetic algorithm and simulated annealing algorithm to search the best phase factors of PTS. The recent scheme proposed by Shukla et.al [77] provides an efficient and optimized search and PAPR performance at low computational complexity for a given value of PAPR threshold. In general, for each data sub-block, the PTS requires V IFFT operations and $V \log _{2} W$ bits as the number of the required side information [42]

\section{Clipping and Filtering}

Clipping is the simplest widely used PAPR reduction technique in OFDM systems. Clipping is mainly performed in the transmitter to limit the maximum of transmit signal to a pre-specified level as well as the parts of the signals that are outside the allowed region [42].Among the disadvantages associated with clipping technique includes, distortion of in-band signal which finally results into BER performance and spectral efficiency degradation[67]. Another drawback is the outof-band interference signals to adjacent channels caused by out-of-band radiation. The out-of band radiation resulted after performing clipping in the transmitter can be reduced by filtering technique at the cost of peak regrowth. However, filtering cannot reduce in-band distortion. In order to reduce peak regrowth, a repeated clipping and filtering operation can be used to obtain a desirable PAPR at a cost of computational complexity increase [67].

Figure 6 shows the block diagram of PAPR reduction in OFDM systems using clipping and filtering technique. Nrepresents the total number of subcarriers where $L$ is the oversampling factor.

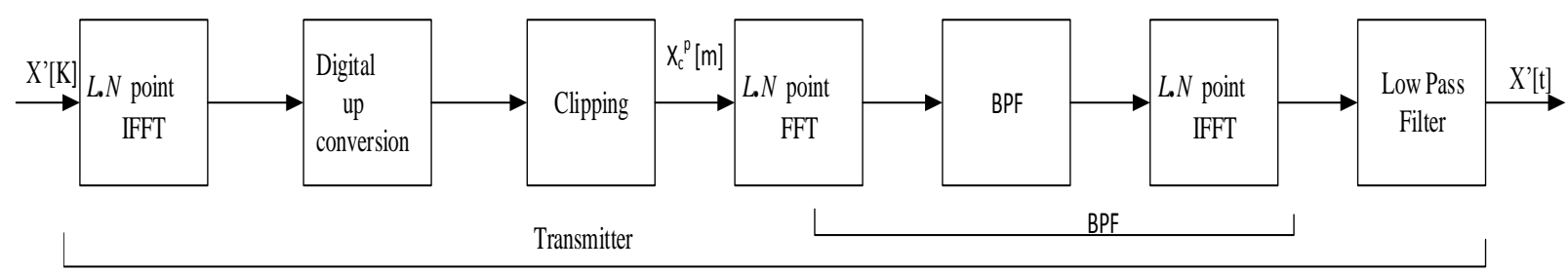

Figure 6: Block Diagram of a PAPR reduction technique using clipping and filtering

$\mathrm{X}_{\mathrm{c}}^{\mathrm{p}}[\mathrm{m}]$ is the clipped pass band signal where $\mathrm{A}$ is a prespecified clipping level.

\section{B CARRIER FREQUENCY OFFSET ESTIMATION AND CORRECTION}

OFDM systems are affected by time and frequency varying wireless channels impairments. Time-varying impairments are caused by the Carrier Frequency Offset (CFO) caused by the mismatch of frequencies between the oscillators at the transmitter and the receiver, or from the doppler spread due to the relative movement between the transmitter and the receiver [3]. Frequency-varying impairments are from the timing offset or the delay spread of wireless channels. Since the duration of the OFDM symbol is longer and its bandwidth is narrower compared with single-carrier modulation, OFDM systems are more robust to frequency-selective fading but more sensitive to time varying impairment of channels. The CFO is divided into two parts namely integerand fractional parts [3][19]. The integer part of the CFO is a multiple of the subchannel space $\Delta f$, which will cause a symbol or subchannel shift when the transmitted symbol in one subchannel is shifted to another at the receiver. The fractional part may cause the loss of orthogonality among subchannels and generates ICI which results into reduction of SNR [19]. When the CFO is estimated, its impact can completely be cancelled in the time domain.

A number of techniques have been proposed for estimating and mitigating the CFO. These techniques are divided into 2 categories namely:

a) Named Data Aided techniques: These techniques are based on the preamble blocks in the time domain or on the pilot sub-channels inserted at different OFDM blocks [78-86].Recently, Salim et.al[86] has proposed an iterative pilot-aided algorithm based on Expectation Conditional Maximization (ECM) for joint estimation of multipath channels, Wiener PHNs, and CFOs in Amplify-and-Forward (AF) based cooperative OFDM systems. The proposed estimator is found to be computationally efficient because it estimates the desired parameters in few iterations and have high SNR. The pilots, training sequences or preambles are basically inserted at the transmitter in order to assist the synchronization at the receiving end [87].

b) Non Data Aided or Blind Techniques: These are non-pilot based methods which exploit the special structure in the OFDM signal, such as virtual sub-channels (VCs) or CP in order to estimate the CFO and do not need an extra overhead [88-93]. 
The performance of these CFO techniques depends on channel features due to the fact that, dispersive fading of wireless channels may destroy the structure of the received signals. In addition, MIMO techniques can also be used in OFDM to form MIMO-OFDM for performance and capacity improvement [3].

\section{THE APPLICATIONS OF OFDM}

OFDM has been adopted in many wireless communication standards and applications such as the new European Digital Audio Broadcasting (DAB)[3][94-97] as well as the Digital terrestrial television Video Broadcasting (DVB)[98], High-definition Television (HDTV) Terrestrial Broadcasting and satellite terrestrial interactive multi-service infrastructure in China[3]. OFDM has been considered and approved by many IEEE standard working groups such as Wireless LAN (IEEE 802.11a/b/g[99] and HiperLAN2).

Multiband OFDM (MB-OFDM) has been used as a candidate for the IEEE 802.15.3a working group designed for ultra-wideband (UWB) systems [100-105].In MBOFDM, the spectrum is divided into several sub-bands where a data stream is transmitted over each band by OFDM[3].In fact, the MB-OFDM for UWB systems can achieve a data rates ranging from 55 to $480 \mathrm{Mb} / \mathrm{s}$ for a distance of up to $10 \mathrm{~m}$.OFDM has been adopted and exploited in the Broadband Wireless Access (BWA) like IEEE 802.16[106], Mobile Broadband Wireless Access (MBWA) like IEEE 802.20[107] and xDSL[19]. OFDMA is one of the most promising radio transmission techniques for LTE of the 3rd Generation Partnership Project(3GPP) and the International Mobile Telecommunications-Advanced Systems(IMT-AS).OFDM -based Wireless Asynchronous Transfer Mode (WATM) transmission system has been proposed in Europe to be suitable for the future broad-band mobile multimedia [14 communications[108]. Cognitive radio has emerged as a promising technology to solve the current spectrum scarcity problem. OFDM can be used to construct the transceiver of cognitive radio by virtue of its flexibility for sub-channel assignment and power allocation [3].

\section{CONCLUSIONS}

The demand for high data rate wireless communication has been increasing dramatically over the last decade. OFDM, which is the most emerging multi-carrier modulation technology of this era, can solve this problem significantly.

This paper has provided a survey of OFDM behaviors, principles and analysed different techniques like Peak-toAverage Power Ratio (PAPR) reduction and frequency offset estimation that improve performance of OFDM for wireless communications. However, other literature shows [21] that OFDM has a strong anti-multi path interference capability in a high-speed data transfer conditions and have also high spectral efficiency. The paper has also explored a brief overview of OFDM applications for wireless communications. Our future work will focus on investigating the performance of different PAPR and CFO techniques through extensive simulations.

Copyright to IJARCCE

\section{REFERENCES}

[1] LIAN Hua, ZhAOruimei, HU boning, PANG Huawei; 2010," Simulation and Analysis of OFDM Communication System", 2010 2nd International Conference on Industrial Mechatronics and Automation. Available in IEEE.

[2] ZHANG jie, L1U liang, LI jin; 2011"Performance Analysis of Space Time Block Code in MIMO-OFDM Systems"Available in IEEE.

[3] Taewon Hwang, Chenyang Yang, Gang Wu, Shaoqian Li, and Geoffrey Ye Li; "OFDM and Its Wireless Applications: A Survey", IEEE TRANSACTIONS ON VEHICULAR TECHNOLOGY, VOL. 58, NO. 4, MAY 2009.

[4] V. Vijayarangan, R. Sukanesh;" 2009,An overview of techniques for reducing peak to average power ratio and its selection criteria for orthogonal frequency division multiplexing radio systems",Journal of Theoretical and Applied Information Technology.

[5] Maan Singh, Vijay Kumar; 2013"Signal Scrambling Techniques for PAPR Reduction in OFDM Systems'International Journal Of Engineering And Computer Science ISSN: 2319-7242 Volume 2 Issue 1 Jan 2013 Page No. 311-317.

[6] Sarika Pal, Avinash Kumar, Narendra Pal;"Performance Analysis of OFDM Mobile Systems for Wireless Communication" Int'1 Conf. on Computer \& Communication Technology ,|ICCCT,2010|,Available in IEEE.

[7] Md. Younus Ali, S. M. ShamsulAlam, Md. Sohel Mahmud Sher, Md.Tariq Hasan, Md. Mizanur Rahman;'Performance Analysis of OFDM in Wireless Communication", 2009 Fourth International Conference on Computer Sciences and Convergence Information Technology.Available in IEEE.

[8] Zhenchuan Zhang, JingjingWu,"Study and Performance Simulation Analysis of OFDMSystems", ICWMMN2006 Proceedings.

[9] ITU-R Report M.2134,Requiremets related to technical performance for IMT-Advanced radio interface(s), Approved in Nov 2008,[Online] available at http://www.itu.int/pub/R-REP-M.2134-2008/en

[10] A.R.S. Bahai, B.R. Saltzberg, multi-carrier digital communications: theory and applications of OFDM, KluwerAcademic/Plenum, Network 1999.

1] B.G. Evan and K. Baughan, "Vision of 4 G," Electronics and Communication Engineering Journal, vol.12, no. 6, pp 293-303, December 2000.

White paper: High-speed wireless OFDM communicationsystems, Wi-LAN Inc., February 2001

13] A. Bello, "Selective fading limitations of the Kathryn modem and some system design considerations," IEEE Trans. Commun.,vol. COM-13, no. 3, pp. 320-333, Sep. 1965.

L. J. Cimini, "Analysis and simulation of a digital mobile channel using orthogonal frequency division multiplexing," IEEE Trans. Commun.,vol. COM-33, no. 7, pp. 665-675, Jul. 1985.

[15] Mosier, R. R., and R. G. Clabaugh, "Kineplex, a Bandwidth Efficient Binary Transmission System," AIEE Trans., Vol. 76, January 1958, pp. 723-728.

[16] Porter, G. C., "Error Distribution and Diversity Performance of a Frequency Differential PSK HF Modem," IEEE Trans. Communications, Vol. COM-16, August 1968, pp.567-575.

[17] Zimmerman, M. S., and A. L. Kirsch, "The AN/GSC-10 (KATHRYN) Variable Rate Data Modem for HF Radio," IEEE Trans. Communications, Vol. COM-15, April 1967, pp.197-205.

[18] P. Howard (2009).MATLAB 7.8 Basics.[Online] available at http://www.math.tamu.edu/ phoward/m442/matbasics.pdf

19] Bo Ai, Chang Yong Pan,Jian Hua Ge,YongWang,Zhe $\mathrm{Lu}(2006)$.On the Synchronization Techniques of Wireless OFDM Systems. Broadcasting, IEEE Transactions on Volume:52, Issue, 2 Page(s) 236 - 244, ISSN 0018-9316.

[20] Wang Yi Gulinfeng .An Investigation of Peak-to-Average Power Reduction in MIMO-OFDM Systems", Blekinge Institute of Technology October 2009.

21] Md. Abdullah Al Baki, Mohammad ZavidParvez "PEAK TO AVERAGE POWER RATIO (PAPR) REDUCTION IN OFDM BASED RADIO SYSTEMS" Electrical Engineering Blekinge Institute of Technology, May 2010.

22] R. W. Bauml, R. F. H. Fischer, and J. B. Huber, "Reducing the peak-to-average power ratio of multicarrier modulation by selected mapping," Electron. Lett., vol. 32, no. 22, pp. 2056-2057, Oct. 1996.

[23] M. Breiling, S. H. Muller, and J. B. Huber, "SLM peak-power reduction without explicit side information,'IEEECommun. Lett., vol. 5, no. 6, pp. 239-241, Jun. 2001. 
[24] C.-L. Wang and Y. Ouyang, "Low-complexity selected mapping [48] schemes for peak-to-average power ratio reduction in OFDM systems," IEEE Trans. Signal Process. vol. 53, no. 12, pp. 4652- [49] 4660, Dec. 2005.

[25] S. H. Muller and J. B. Huber, "OFDM with reduced peak-toaverage power ratio by optimum combination of partial transmit [50] sequences," Electron. Lett., vol. 33, no. 5, pp. 368-369, Feb. 1997.

[26] Y. Xiao, X. Lei, Q. Wen, and S. Li G., "A class of low complexity PTS techniques for PAPR reduction in OFDM systems," IEEE Signal Process. Lett., vol. 14, pp. 680-683, Oct. 2007.

[27] S.-J. Ku, C.-L. Wang, and C.-H. Chen, "A reduced-complexity PTS-based PAPR reduction scheme for OFDM systems," IEEE Trans. Wireless Commun., vol. 9, no. 8, pp. 2455-2460, Aug. 2010.

[28] J.-C. Chen, "Partial transmit sequences for peak-to-average power ratio reduction of OFDM signals with the cross-entropy method," IEEE Signal Process. Lett., vol. 16, no. 6, pp. 545-548, Jun. 2009.

[29] Y.-J. Kou, W.-S. Lu, and A. Antoniou, "A new peak-to-average power ratio reduction algorithm for OFDM systems via [53] constellation extension," IEEE Trans. Wireless Commun., vol. 6, no. 5, pp. 1823-1832, May 2007.

[30] C.-L. Wang, Y.-C. Tsai, and S.-J. Ku, "A low-complexity constellation extension scheme for PAPR reduction of OFDM signals," in Proc. 2009 IEEE Veh. Technol. Conf.-Fall (VTC 2009- [ 55 ] Fall), Anchorage, Alaska, Sep. 2009

[31] Han, S.H. and Lee, J.H. (2005) An overview of peak-to-average [56] power ratio reduction techniques for multicarrier Transmission. IEEE Wireless Commun., 12(2), 56-65.

[32] Wilkinson, T.A. and Jones, A.E. (July 1995) Minimization of the peak-to-mean envelope power ratio of multicarrier transmission scheme by block coding. IEEE VTC'95, Chicago, vol. 2, pp. 825-829.

[33] Golay, M.J.E. (1961) Complementary series. IEEE Trans. Inf. [58] Theory, 7(2), 82-87.

[34] Popovic, B.M. (1991) Synthesis of power efficient multitone signals with flat amplitude spectrum. IEEE Trans. Commun., 39(7), 1031-1033.

[35] Van Nee, R.D.J. (Nov. 1996) OFDM codes for peak-to-average power reduction and error correction. IEEE GTC, London, vol. 2, pp. 740-744.

[36] 179. Davis, J.A. and Jedwab, J. (1999) Peak-to-mean power control [60] in OFDM, Golay complementary sequences, and Reed-Muller codes. IEEE Trans. Info. Theory, 45(7), 2397-2417.

[37] 180. Davis, J.A. and Jedwab, J. (1997) Peak-to-mean power control [61] and error correction forOFDMtransmission using Golay sequences and Reed-Muller codes. Electron. Lett., 33(4), 267-268.

[38] Urbanke, R. and Krishnakumar, A.S. (Oct. 1996) Compact description of Golay sequences and their extensions. Proc. of the [62] Thirty-Fourth Annual Allerton Conference on Commun., Control and Computing Pagination, Urbana, IL, pp. 693-701.

[39] Li, X. and Ritcey, J.A. (1997) M-sequences for OFDM peak-to- [63] average power ratio reduction and error correction. Electron. Lett., 33(7), 554-555.

[40] Tellambura, C. (1997) Use of m-sequences for OFDM peak-to- [64] average power ratio reduction. Electron. Lett.,33(15), 1300-1301.

[41] Park, M.H. et al. (2000) PAPR reduction inOFDMtransmission [65 using Hadamard transform. IEEE ICC'00, vol. 1,pp. 430-433.

[42] Yong Soo Cho, Jaekwon Kim, Won Young Yang, Chung G. Kang (2010). MIMO-OFDM Wireless Communications with Matlab. John Wiley \& Sons (Asia) Pte Ltd, 2 Clementi Loop, \# 02-01, Singapore 129809, ISBN 978-0-470-82561-7

[43] V. Vijayarangan and R. Sukanesh, "An overview of techniques for reducing peak to average power ratio and its selection criteria for orthogonal frequency division multiplexing radio systems," Journal of Theoretical and Applied Information Technology, vol. 5, no. 1, pp. 25-36, 2005.

[44] May, T., and Rohling, H., "Reducing the peak to average power ratio of OFDM", radio transmission system", IEEE Vehicular Technology Conference, Vol. 3, May 1998.

[45] Byung Moo Lee,Youngok Kim, An Adaptive Clipping and Filtering Technique for PAPR Reduction of OFDM Signals, Circuits, Systems, and Signal ProcessingJune 2013, Volume 32, Issue 3, pp 1335-1349.

[46] J. Armstrong, Peak-to-average power reduction for OFDM by repeated clipping and frequency domain filtering. Electron. Lett. 38(5), 246-247 (2002).

[47] Y.-J. Kou, W.-S. Lu, and A. Antoniou, "A new peak-to-average power ratio reduction algorithm for OFDM systems via constellation extension," IEEE Trans. Wireless Communications., vol. 6, no. 5, pp. 1823-1832, May 2007.
R. Gross and D. Veeneman, "Clipping distortion in DMT ADSL systems," Electron. Lett., vol. 29, no. 24, pp.2080-2081, Nov. 1993. Van Nee, R., and Wild, A., "Reducing the peak to average power ratio of OFDM", IEEE Vehicular Technology Conference, Vol.3, pp. 18-21 may 1998.

Foomooljareon, P. and Fernando, W.A.C., "Input sequence envelope scaling in PAPR reduction of OFDM", IEEE 5th international symposium on wireless personal multimedia communications, Vol.1, Oct 2002.

51] Nikookar, H., and Lidsheim, k.S., "Random phase updating algorithm for OFDM transmission with low PAPR", IEEETransaction on Broadcasting, Vol.48, Jun 2002.

2] Tan, C.E. Wassell, I.J., "Data bearing peak reduction carriers for OFDM systems", IEEE Proceedings of the 2003 Joint Conference of the Fourth International Conference of Information, Communications and Signal Processing, 2003 and the Fourth Pacific Rim Conference on Multimedia, Vol.2, Dec 2003.

53] Telladoj.' "Peak to average power reduction for multicarrier modulation", Ph.D. distortion, standard University 2000

4] Wang, X., Tjhung, T.T., Ng, C.S., "Reduction of peak to average power ratio of OFDM system using a companding technique", IEEE transactions on broadcasting, Vol.25, Sep 1999.

Li, X. and Cimini, L.J. (1998) Effects of clipping and filtering on the performance of OFDM. IEEE Commun. Letter, 2(20), 131-133.

Slimane, S.B. (Dec. 2000) Peak-to-average power ratio reduction of OFDM signals using pulse shaping. IEEE GTC, vol. 3, pp. 1412-1416.

57] C.-L. Wang, S.-S. Wang, and S.-L. Chang, "A low-complexity SLM based PAPR reduction scheme for SFBC MIMO-OFDM systems," in Proc. 2011 IEEE Wireless Commun. \& Network. Conf. (WCNC 2011), Cancun, Mexico, Mar. 2011

Sheng-JuKu(2014) A Low-Complexity PTS Scheme for PAPR Reduction in SFBC MIMO-OFDM Systems; 978-1-4799-4449-1/14, 2014 IEEE. ShenghuaZhai(2014). Flexible Nonlinear Companding Transform for Reducing PAPR of Multi-carrier Signals, 2014 IEEE International Conference on Computer and Information Technology,IEEE Computer Society.

Jeon, W.G., Chang, K.H., and Cho, Y.S. (1997) An adaptive data predistorter for compensation of nonlinear distortion in OFDM systems. IEEE Trans. on Commun., 45(10), 1167-1171.

M. Mroue, A. Nafkha, J. Palicot, Gavalda B., and N. Dagorne, "Performance and implementation evaluation of TR PAPR reduction methods for DVB-T2," International Journal of Digital Multimedia Broadcasting, vol. 2010, pp. 2-4, Apr. 2010.

Jayalath, A.D.S., and Tellambura, C., "The use of interleaving to reduce the peak to average power ratio of an OFDM signals", IEEE Global telecommunications conference, Vol.1, Nov 2000.

Bruninghaus, K. and Rohling, H. (May 1998) Multi-carrier spread spectrum and its relationship to single-carrier transmission. IEEE VTC'98, vol. 3, pp. 2329-2332.

Galda, D. and Rohling, H. (2002)A low complexity transmitter structure forOFDM-FDMAuplink systems. IEEE VTC'02, vol. 4, pp. 1737-1741.

Myung, H.G., Lim, J., and Goodman, D.J. (2006) Single carrier FDMA for uplink wireless transmission. IEEE Veh. Technol. Mag., 1(3), 30-38.

[66] Myung, H.G., Lim, J., and Goodman, D.J. (Sept. 2006) Peak-toaverage power ratio of single carrier FDMA signals with pulse shaping. PIMRC'06, pp. 1-5.

[67] Tao Jiang and Yiyan Wu, An Overview: Peak-to-Average Power Ratio Reduction Techniques for OFDM Signals; IEEE TRANSACTIONS ON BROADCASTING, VOL. 54, NO. 2, JUNE 2008.

[68] S. H. Han and J. H. Lee, "PAPR reduction of OFDM signals using a reduced complexity PTS technique," IEEE Signal Processing Letters, vol. 11, no. 11, pp. 887-890, Nov. 2004.

[69] X. Y. Zhang and G. B. Tao, "The research of improved PTS method for peak-to-average power ratio reduction," Proceedings of IEEE $3^{\text {rd }}$ international conference on wireless, mobile and multimedia networks, Beijing, China, pp.104-107, September 2010.

70] P. Mukunthan and P. Dananjayan, "PAPR Reduction by Modified PTS Combined with Interleaving Technique for OFDM System with QPSK Subcarriers," IEEE International Conference on Advances in Engineering, Science and Management, pp.410-415, March 2012.

L. Yang, R. S. Chen, Y. M. Siu, and K. K. Soo,"PAPR reduction of an OFDM signal by use of PTS with low computational complexity," IEEE Trans. Broadcast, vol. 52, pp. 83-86, March 2006.

S. S. Kim, M. J. Kim and T. A. Gulliver, "PAPR Reduction of OFDM Signals Using Genetic Algorithm PTS Technique," IEICE Trans. Communication, vol. E91-B, no.4, pp. 1194-1197, April 2008. 
[73] H. Y. Liang, Y. R. Chen, Y.F. Huang, and C. H. Cheng, “A Modified [94] Genetic Algorithm PTS Technique for PAPR Reduction in OFDM [95] Systems," Proceedings of the 15th Asia-Pacific Conference on Communications, pp.182-185, 2009

[74] Xia Wang, Songhua He, Tao Zhu.” A Genetic-Simulated Annealing Algorithm Based On PTS Technique for PAPR Reduction in OFDM System, 2014 Symposium on Computer Applications and Communications. IEE Computer Society.

[75] Shameema Hameed L, "A Combined Weighting and PTS Technique for PAPR Reduction in OFDM Signals", 2 nd International Conference on Current Trends in Engineering and Technology, ICCTET'14, IEEE Conference Number - 33344 July 8, 2014, Coimbatore, India

[76] Zeyid T. Ibraheem, Md. Mijanur Rahman, S. N. Yaakob, Rasim A. Kadhim, Kawakib K. Ahmed, Mohammad ShahrazelRazalli, Performance of PTS Techniques with Varied Partition Size in PAPR Reduction of OFDM System, 2014 IEEE 2014 International Conference on Computer, Communication, and Control Technology (I4CT 2014), September 2-4, 2014 - Langkawi, Kedah, Malaysia.

[77] Jyoti Shukla, A lok Joshi, Rahgav Bansal, R.K.Tyagi.” PAPR Reduction of OFDM systems Using PTS withGenetic Algorithm at low computational complexity",IEEE International Conference on [101] Recent Advances and Innovations in Engineering (ICRAIE-2014), May 09-11, 2014, Jaipur, India.

[78] T. M. Schmidl and D. C. Cox, "Robust frequency and timing synchronization for OFDM,” IEEE Trans. Commun., vol. 45, no. [102] 12, pp. 1613-1621, Dec. 1997.

[79] Amine Laourine, Alex Stephenne and SofieneAffes, A new data- [103] aided carrier frequency offset estimation algorithm for OFDM systems,IEEE 2006.

[80] E. C. Kim, J. Yang and J. Y. Kim, "Novel of DM Frame Synchronization and Frequency Offset Compensation Scheme for [104] Wireless Multimedia Communication Sys-tems," IEEE Transactions on Consumer Electronics, Vol. 55, 2009, pp. 11411148. doi:10.1109/TCE.2009.5277968

[81] A. B. Awoseyila, C. Kasparis and B. G. Evans, "Robust Timedomain Timing and Frequency Synchronization for OFDM Systems," IEEE Transactions on Consumer Elec-tronics, Vol. 55, 2009, pp. 391-399.

[82] I. Kim, Y. Han and H. K. Chung, "An Efficient Synchro-nization Signal Structure for OFDM-Based Cellular Sys-tems," IEEE Transactions on Wireless Communications, Vol. 9, 2010, pp. 99 105. doi:10.1109/TWC.2010.01.090516

[83] J. W. Choi, ,J. W. Lee, Q. Zhao and H. L. Lou, “Joint ML Estimation of Frame Timing and Carrier Frequency Off-set for OFDM Systems employing Time-Domain Re-peated Preamble," IEEE Transactions on Wireless Com-munications, Vol. 9, 2010, pp. [108] 311- 317. doi:10.1109/TWC.2010.01.090674

[84] C. L. Wang and H. C. Wang, "Large CFO Acquisition using Partial Geometric Modulatable Orthogonal Se-quences," IEEE VTC Spring , 2009, pp. 1-5.

[85] W. Zhong, "A Simple and Robust Estimation of Integral Frequency [109] Offset for OFDM Systems," IEEE Transac-tions on Consumer Electronics Vol. 54, 2008, pp. 411- 413.doi:10.1109/TCE. 2008.4560107

[86] Omar H. Salim, Ali A. Nasir, Wei Xiang and Rodney A. Kennedy."Joint Channel, Phase Noise, and Carrier Frequency Offset Estimation in Cooperative OFDM Systems",IEEE ICC 2014 - Signal Processing for Communications Symposium.

[87] S. Younis, A. Al-Dweik1, C. C. Tsimenidis1, B. S. Sharif1 and A. Hazmi(2011), The Effect of Timing Errors on Frequency Offset Estimation in OFDM Systems,IEEE,2011.

[88] J. van de Beek, M. Sandell, and P. Borjesson, "ML estimation of timing and frequency offset in OFDM systems," IEEE Trans. Signal Proc., vol.45, pp. 1800-1805, Jul. 1997

[89] Y. Yingwei and G. B. Giannakis, "Blind carrier frequency offset estimation in SISO, MIMO, and multiuser OFDM systems," IEEE Trans. Commun., vol. 53, pp. 173-183, Jan. 2005.

[90] A. J. Al-Dweik, "Robust non data-aided frequency offset estimation technique," in Proc. IEEE PIMRC, 2004, pp. 1365-1369.

[91] Z. Xiang and A. Ghrayeb, "A blind carrier frequency offset estimation scheme for OFDM systems with constant modulus signaling," IEEE Trans. Commun., vol. 56, pp. 1032-1037, 2008.

[92] A. Al-Dweik, A. Hazmi, S. Younis, B. Sharif, and C. Tsimenidis, "Carrier frequency offset estimation for OFDM systems over mobile radio channels," IEEE Trans. Veh. Technol., vol. 59, pp. 974 - 979, Feb. 2010.

[93] A. J. Al-Dweik," A Novel Non-Data-Aided Symbol Timing Recovery Technique for OFDM Systems", IEEE TRANSACTIONS ON COMMUNICATIONS, VOL. 54, NO. 1, JANUARY 2006
DAB," in Proc. 1st Int. Symp., Montreux, Switzerland, June 1992. F. Mueller-Roemer, "Directions in audio broadcasting," J. Audio Eng. Soc., vol. 41, no. 3, pp. 158-173, Mar. 1993.

[96] G. Plenge, "DAB-A new radio broadcasting system-State of development and ways for its introduction," Rundfunktech. Mitt, vol.35, no. 2 , p. 45,1991

M. Alard and R. Lassalle, "Principles of modulation and channel coding for digital broadcasting for mobile receivers," $E B U$ Review, pp. 47-69, Aug. 1987. Tech. 224 “

[98] ETSI, "Digital video broadcasting (DVB); framing structure, channel coding and modulation for digital terrestrial television," EN 300744 V1.1.2, Aug. 1997

[99] IEEE P802.11a/D7.0, “ DRAFT Suplement to STANDARD for Information Technology Telecommunications and information exchange between systems - Local and metropolitan area networks - Specific Requirements - Part II Wireless LAN Medium Access Control (MAC) and Physical Layer (PHY) specifications: High Speed Physical Layer in the 5GHz Band," July 1999 IEEE 802.15 WPAN High Rate Alternative PHY Task Group $3 a$ (TG3a). [Online]. Available: www.IEEE802.org/15/pub/TG3a.html

W. P. Siriwongpairat, W. Su, M. Olfat, and K. J. R. Liu, "Multiband- OFDM MIMO coding framework for UWB communication systems," IEEE Trans. Signal Process. vol. 54, no. 1, pp. 214-224, Jan. 2006.

A. Batraet al., Multiband OFDM Physical Layer Specification. San Ramon, CA: WiMedia Alliance, Jul. 2005. Rel. 1.1.

J. Foersteret al., "Channel modeling sub-committee report final," IEEE P802.15 Working Group for Wireless Personal Area Networks (WPANs), Oct. 2005. IEEE P802.15-02/490r1-SG3a.

B.-W. Koo, M.-S. Baek, Y.-H. You, and H.-K. Song, "Highspeed MB-OFDM system with multiple antennas for multimedia communication and home network," IEEE Trans. Consum. Electron., vol. 52, no. 3, pp. 844-849, Aug. 2006

[105] I. R. Capoglu, Y. (G.) Li, and A. Swami, "Effect of Doppler spread in OFDM-based UWB systems," IEEE Trans. Wireless Commun., vol. 4,no. 5, pp. 2559-2567, Sep. 2005.

[106] "IEEE P802.16, Part 16: Air interface for fixed broadband wireless access systems," Revision of IEEE Std. 802.16-2004 as amended by IEEE Std. 802.16f-2005 and IEEE Std. 802.16e2005, Mar. 2007. Draft.

[107] "IEEE 802.20: Mobile Broadband Wireless Access (MBWA)". Official standards free download web page. IEEE 802 committee. Retrieved August 20, 2011

J. Borowski, S. Zeisberg, J. Hübner, K. Koora, E. Bogenfeld, and B. Kull, "Performance of OFDM and comparable single carrier system in MEDIAN demonstrator $60 \mathrm{GHz}$ channel," in Proc. ACTS Summit, Aalborg, Denmark, Oct. 1997, pp. 653-658. http://wiki.china.org.cn/wiki/index.php/4G 